\title{
The signaling effect of raising inflation*
}

\author{
Jean Barthélemy $\quad$ Eric Mengus
}

February 15, 2016

\section{PRELIMINARY}

\begin{abstract}
This paper argues that central bankers can raise inflation to signal their ability to commit to forward guidance policies. As inflation can be stabilized in normal times either because of central banker's commitment ability or because of his aversion to inflation, the private sector is unable to infer the central banker's type from observing stable inflation before a liquidity trap, jeopardizing the efficiency of forward guidance policy. We derive optimal policy in a new-Keynesian model subject to liquidity traps where agents are uncertain about the central banker's type and we show that the central banker with commitment ability can signal its type by raising inflation before a trap. The corresponding level of signaling inflation increases with the frequency, the severity as well as with the length of liquidity traps. Finally, we show that this signaling motive can explain level of inflation well above $2 \%$.
\end{abstract}

Keywords: Forward Guidance, Inflation, Signaling.

JEL Classification: E31, E52, E65.

*Barthélemy: Sciences Po, Department of Economics, 28 rue des Saints Pères 75007 Paris, France and Banque de France, 31 rue Croix des Petits-Champs 75049 PARIS cedex 01. Email: jean.barthelemy@ sciences-po.fr. Mengus: HEC Paris, Economics and Decision Sciences Department, 1 rue de la Libération, 78350 Jouy-en-Josas, France. Email: mengus@hec.fr. We thank Philippe Andrade, Nicolas Coeurdacier, Gaetano Gaballo, Serguei Guriev, Emeric Henry, Jean Tirole and François Velde for helpful comments as well as seminar participants at Sciences Po. All remaining errors are ours. 


\section{Introduction}

When nominal interest rates hit the zero lower bound (ZLB, thereafter), monetary policy can still accommodate for deflationary shocks by committing to keep interest rates low, even after the shock disappears - the so-called forward guidance policy. However, as argued by Eggertsson and Woodford (2003) or Werning (2012), forward guidance is subject to timeinconsistency as, depending on his preferences, the central banker may have incentives to raise interest rates ex-post. Forward guidance's efficiency may thus be limited if private agents doubt the central banker's commitment ability.

In this paper, we study optimal monetary policy when private agents are uncertain about the central banker's preferences and we determine whether standard monetary policy instruments can be used to signal commitment ability. Our main result is that raising inflation before a liquidity trap occurs can achieve such a signaling so that private agents anticipate a forward guidance policy once the economy actually falls into a liquidity trap.

But why signal through higher inflation? Forward guidance policy requires that the central banker commits to a future inflationary boom. Such a boom is least likely to be engineered by central bankers who are highly averse to inflation - such as the conservative central banker who only aims at stabilizing inflation. ${ }^{1}$ And so, the central banker who can commit has every incentive to be distinguished from such conservative central bankers. And because conservative central bankers are inclined to stabilize inflation in normal times, increasing inflation ex ante is the only available tool to achieve such a separating.

To formalize that argument, we consider a standard new Keynesian model facing shocks on the natural rate of interest that push the economy to a liquidity trap. In contrast with the literature, the private sector cannot observe the central banker's preferences for output-gap stabilization. Indeed, an intermediate weight on output-gap stabilization in central banker's preferences is necessary to achieve the Ramsey allocation when introducing reputation concerns. In the model, we first limit the central banker's types to two: either he maximizes welfare (the benevolent central banker) or he only takes inflation into account in his objective function and hence does not implement forward guidance (the conservative central banker). We then consider the perfect Bayesian equilibrium of this economy and we find the following results.

Our main insight is that inflation before the liquidity trap occurs can be used as a signaling device for the benevolent central banker. Indeed, as inflation is the only variable entering into the conservative central banker's objective function, it is the means to distinguish between the

\footnotetext{
${ }^{1}$ See Rogoff (1985) and the literature on delegating monetary policy.
} 
benevolent central banker and the conservative one as the latter will not be inclined to mimic the former. The benevolent central banker then optimally sets inflation at the rate at which the conservative central banker would break even between mimicking the benevolent central banker and adopting a conservative monetary policy. This inflation rate increases with the probability, the intensity and the length of liquidity traps.

Furthermore, signaling is possible only if the benevolent central banker has some inflation bias. Even though this inflation bias does not affect the equilibrium outcome, it makes inflation relatively less costly for him than for the conservative central banker. ${ }^{2}$

Turning to quantification, the required inflation level is significantly higher than 0 . When calibrating our model, we show that this signaling motive can generate a level of inflation of around $2 \%$. In addition, we observe that even small movements in the probability of ZLB or an increase in the expected length of the trap can increase the level of signaling inflation to above $3 \%$. Note that, in our framework, inflation does not reduce the probability of a liquidity trap and does not help to reduce the consequences of this liquidity trap, so that these values only reflect our signaling motive. ${ }^{3}$

Finally we extend our signaling problem to the case where the benevolent central banker can be mistaken to be an inflation-biased discretionary central banker as well as a conservative one. In this context, we show that our main result is robust: benevolent central bankers need to implement inflation before a trap occurs, even if this implies to be pooled with the inflationbiased central banker.

Related literature. Raising inflation has been suggested as a possible policy to avoid liquidity traps (see Blanchard et al., 2010, among others). By widening the size of shocks required to hit the zero lower bound on nominal interest rates, inflation can act as a buffer. This proposal has also been defended by Ball (2014) and the Fed's president Rosengren (April 16,2015), who argue that the cost of liquidity traps is large, policies used to escape the trap are inefficient and the probability of a liquidity trap has increased. Aruoba and Schorfheide (2013) show that recovery from the Great Recession in the US would have been a year shorter if the Fed had targeted $4 \%$ inflation instead of the observed 2.5\%. Yet, from a normative standpoint, the optimal size of this buffer has been found to be quantitatively small as liquidity traps are nevertheless rare events (see Coibion et al., 2012). In this paper, we investigate an alternative

\footnotetext{
${ }^{2}$ In other words, the inflation bias in benevolent central bankers' preferences plays the role of a SpenceMirrlees condition in the signaling problem.

${ }^{3}$ This explains why we find different values compared with Coibion et al. (2012) who consider the buffer motive of raising inflation.
} 
motive for raising inflation in the presence of ZLB, where inflation acts as a signal of the central banker's willingness to implement forward guidance policies.

Bassetto (2015) analyses communication on forward guidance policies. Our view is that hard information through signaling inflation is consistent with his insight that pure communication on commitment is cheap talk. Yet, in our case, communicating about preferences (the inflation bias), as it is connected with the commitment ability, would not affect the set of achievable outcomes for central bankers: announcing inflation bias, and thus commitment ability, is also cheap talk in our case.

Our paper is also connected to the literature on imperfect credibility at the ZLB as in Bodenstein et al. (2012). The main difference is that imperfect credibility in our framework is an equilibrium outcome that can be altered by a monetary authority's actions. In addition, Andrade et al. (2015) provide evidence on heterogeneous understanding of forward guidance policies and link this to imperfect credibility of the central bank.

In addition to the papers that we have already mentioned, our paper is closely related to the literature on learning on central bankers' types as in Backus and Driffill (1985) or Barro (1986) (see also Sleet and Yeltekin (2007) for a recent contribution). Our paper's main difference is that we introduce the possibility of liquidity traps and also we allow the central banker under commitment to optimally react to mimicking strategies by a discretionary central banker.

\section{Model under perfect information}

In this section, we introduce the standard new-Keynesian model in the presence of a zero lower bound as in Eggertsson and Woodford (2003) and Werning (2012). Our main assumptions concern the process of the natural rate of interest, that may push the economy at the ZLB to allow for tractability. We relax most of these assumptions once we quantify the signaling mechanism.

\section{$2.1 \quad$ Model}

Time is discrete and denoted by $t \in\{-\infty, \ldots \infty\}$. We consider an economy summarized by two equations:

$$
\begin{aligned}
\pi_{t} & =\beta E_{t} \pi_{t+1}+\kappa y_{t}, \\
y_{t} & =E_{t} y_{t+1}-\frac{1}{\sigma}\left(i_{t}-E_{t} \pi_{t+1}-r_{t}^{n}\right) .
\end{aligned}
$$

where $\pi_{t}$ denotes (log of) date-t inflation rate, $y_{t}$ the output gap, $i_{t}$ the nominal interest rate and $r_{t}^{n}$ the natural rate of interest. Scalars $\sigma, \beta$ and $\kappa$ are positive constants. Finally, $E_{t}$ 
denotes the time-t expectations operator.

The New Keynesian Phillips curve (NKPC) describes the dynamics of prices. The Euler equation (EE) derives from the consumer's inter temporal consumption choice.

Finally, the Zero Lower Bound on nominal interest rate imposes:

$$
i_{t} \geq-\bar{i}, \text { with } \bar{i} \geq 0
$$

Assumption 1 (Process). The natural rate of interest $\left(r_{t}^{n}\right)$ is 0 with probability $1-\gamma$ and $-r$ otherwise. Once the natural rate of interest equals $-r$, it returns to 0 next period and for any subsequent period. We assume that $-r$ is sufficiently low.

This assumption is convenient for deriving analytical results but involves no loss of generality. We depart from this assumption in the numerical application, where we consider a liquidity trap lasting more than one period. We also discuss the role of possible future liquidity traps in Section 7.

Welfare We suppose that the date-s economy's welfare is given by the opposite of the following quadratic loss function: ${ }^{4}$

$$
L_{s}=\frac{1}{2} E_{s} \sum_{t=s}^{\infty} \beta^{t-s}\left[\pi_{t}^{2}+\lambda\left(y_{t}-y^{*}\right)^{2}\right],
$$

where the scalar $y^{*} \geq 0$ denotes the desired level of the output gap, also referred to as the inflation bias, and the scalar $\lambda \geq 0$ denotes the weight of output fluctuations in the welfare function.

For deriving analytical results, we restrict the policy parameter, $\lambda$ as follows:

Assumption 2 (Output gap weight). The weight on the output-gap in the welfare-relevant loss function is sufficiently high, i.e. $\sigma \kappa<\lambda$.

When this condition is satisfied, the optimal monetary policy under commitment leads to a forward guidance type of policy similar to Eggertsson and Woodford (2003) characterized by a positive inflation rate and output-gap after the liquidity trap. More about this assumption can be found in Werning (2012). In the numerical applications, we relax this assumption.

Central bankers We consider a continuum of central bankers indexed by $\lambda_{D}$, so that the $\lambda_{D}$-central banker's preference is:

$$
L_{s}^{\lambda_{D}}=\frac{1}{2} E_{s} \sum_{t=s}^{\infty} \beta^{t-s}\left[\pi_{t}^{2}+\lambda_{D}\left(y_{t}-y^{*}\right)^{2}\right]
$$

\footnotetext{
${ }^{4} \mathrm{~A}$ micro-foundation of this loss can be found in Woodford (2003).
} 
When the preference parameter, $\lambda_{D}$, is equal to zero we call the central banker the conservative central banker. When this parameter is equal to $\lambda$, we call him the benevolent central banker as he maximizes the welfare function.

\subsection{Optimal policies under full information}

Let us first describe the optimal monetary policy, i.e. the one adopted by the benevolent central banker under commitment, in this economy. Then we compute optimal policies of a $\lambda_{D}$-central banker under discretion. Finally, we derive the policy implemented by central bankers in the presence of reputation concerns. In all this subsection, we suppose that the central banker's type, indexed by $\lambda_{D}$, is common knowledge.

The Ramsey allocation. Under commitment, the benevolent central banker maximizes date-s welfare with respect to the entire sequence of nominal interest $\left\{i_{t}\right\}_{t \geq s}$, given that the allocation solves equations (NKPC),(EE) and (ZLB). Date $s$ is supposed to be in the distant past $(s \rightarrow-\infty)$ to allow for a stationary equilibrium before the liquidity trap. We call the resulting outcome the Ramsey allocation. Formally, the central banker solves the following problem:

Problem 1 (Ramsey program).

$$
\min _{i_{t}, t \geq s} L_{s}
$$

under the constraints (NKPC), (EE) and (ZLB).

The Lagrangian of this problem can be written as:

$$
\begin{aligned}
E_{s} \sum_{t=s}^{\infty} \beta^{t-s} & {\left[\frac{1}{2}\left(\pi_{t}^{2}+\lambda\left(\hat{y}_{t}-y^{*}\right)^{2}\right)+\mu_{t}\left(\pi_{t}-\beta E_{t} \pi_{t+1}-\kappa \hat{y}_{t}\right)\right.} \\
+ & \left.\nu_{t}\left(y_{t}-E_{t} y_{t+1}+\frac{1}{\sigma}\left(i_{t}-E_{t} \pi_{t+1}-r_{t}^{n}\right)\right)+\zeta_{t}\left(i_{t}+\bar{i}\right)\right],
\end{aligned}
$$

where $\mu_{t}, \nu_{t}$ and $\zeta_{t}$ are the Lagrange multipliers associated to the constraints (NKPC), (EE) and (ZLB). This latter constraint being an inequality, the Lagrange multiplier $\zeta_{t}$ is strictly negative if the constraint is slack, zero otherwise. First order conditions lead to:

$$
\begin{aligned}
& \pi_{t}+\mu_{t}-\mu_{t-1}-\frac{\nu_{t-1}}{\beta \sigma}=0 \\
& \lambda\left(y_{t}-y^{*}\right)-\kappa \mu_{t}+\nu_{t}-\frac{1}{\beta} \nu_{t-1}=0 \\
& \frac{1}{\sigma} \nu_{t}+\zeta_{t}=0
\end{aligned}
$$


The Ramsey allocation is the solution of the system defined by first-order conditions and equations (NKPC), (EE) and (ZLB).

Prior to the trap, as the date $s$ is supposed to be in the distant past, ${ }^{5}$ inflation is fully stabilized $\left(\pi_{t}=0\right)$. However, the expectation of a liquidity trap triggers a positive output gap, $-\frac{\beta \gamma}{\kappa} \pi_{l}$, where $\pi_{l}$ is the rate of inflation at the ZLB. After the trap, the Ramsey allocation can be implemented through forward Guidance policy associated with positive inflation, $\pi_{l+1}$, and output gap, $y_{l+1}$, at the end of the trap. Afterward, both inflation and output gap converge to 0 .

$\lambda_{D}$-central banker under discretion Under full information and under discretion, the $\lambda_{D}$-central banker solves at each period the following optimization program:

Problem 2 (Under discretion).

$$
\forall t, \min _{i_{t}}\left[\pi_{t}^{2}+\lambda_{D}\left(y_{t}-y^{*}\right)^{2}\right]
$$

under the constraints (NKPC), (EE) and (ZLB).

The Lagrangian writes:

$$
\begin{aligned}
& 1 / 2\left[\pi_{t}^{2}+\lambda_{D}\left(y_{t}-y^{*}\right)^{2}\right]+\ldots \\
& \ldots .+\mu_{t}\left(\pi_{t}-\beta E_{t} \pi_{t+1}-\kappa y_{t}\right)+\nu_{t}\left(y_{t}-E_{t} y_{t+1}+\frac{1}{\sigma}\left(i_{t}-E_{t} \pi_{t+1}-r_{t}^{n}\right)\right)+\zeta_{t}\left(i_{t}+\bar{i}\right),
\end{aligned}
$$

and the resulting first order conditions lead to:

$$
\kappa \pi_{t}+\lambda_{D}\left(y_{t}-y^{*}\right)=\sigma \zeta_{t}
$$

where $\zeta_{t}$ stands for the Lagrange multiplier associated with the constraint (ZLB). As a result, inflation and the output gap follow:

In any period after the liquidity trap:

$$
\pi_{t}=\lambda_{D} \frac{\kappa y^{*}}{\kappa^{2}+\lambda_{D}(1-\beta)} \text { and } y_{t}=\lambda_{D} \frac{(1-\beta) y^{*}}{\kappa^{2}+\lambda_{D}(1-\beta)},
$$

During the liquidity trap:

$$
\pi_{t}=\kappa\left[\lambda_{D} y^{*} \frac{1+\frac{\kappa}{\sigma}}{\kappa^{2}+\lambda_{D}(1-\beta)}-\frac{1}{\sigma}(\bar{i}-r)\right] \text { and } y_{t}=\lambda_{D} y^{*} \frac{(1-\beta)+\frac{\kappa}{\sigma}}{\kappa^{2}+\lambda_{D}(1-\beta)}-\frac{1}{\sigma}(\bar{i}-r),
$$

\footnotetext{
${ }^{5}$ This assumption is sometimes referred to as optimal policy in a timeless perspective, see Woodford (2003)
} 
Before the liquidity trap:

$$
\pi_{t}=\lambda_{D} \frac{\beta \gamma \pi_{l}+\kappa y^{*}}{\kappa^{2}+(1-\beta(1-\gamma)) \lambda_{D}} \text { and } y_{t}=\frac{(1-\beta(1-\gamma)) \lambda_{D} y^{*}-\kappa \beta \gamma \pi_{l}}{\kappa^{2}+(1-\beta(1-\gamma)) \lambda_{D}} .
$$

In particular, note that inflation before the trap equals 0 whatever the probability of ZLB $(\gamma)$ if and only if $\lambda_{D}$ equals zero. ${ }^{6}$

Sustainable policies. We now investigate whether a $\lambda_{D}$-type central banker can sustain the Ramsey allocation under a trigger strategy in the spirit of Chari and Kehoe (1990). The resulting sustainability condition is:

$$
\sum_{k=t}^{\infty} \beta^{t-k}\left(\pi_{k}^{2}+\lambda_{D}\left(y_{k}-y^{*}\right)^{2}\right) \leq U_{t}^{d}+W^{D},
$$

where $U^{d}$ is the current gain from the best deviation given private agents' expectations consistent with the Ramsey allocation and $W^{D}$ is the present value of future periods' losses under discretion - which is the worst equilibrium and therefore the largest punishment compatible with a competitive equilibrium. Using the results from previous paragraphs, this condition can be rewritten when the ZLB is not binding as follows:

$$
\sum_{k=t}^{\infty} \beta^{t-k}\left(\pi_{k}^{2}+\lambda_{D}\left(y_{t}-y^{*}\right)^{2}\right) \leq\left(\frac{\left(\kappa y^{*}+\beta E_{t} \pi_{t+1}\right)^{2}}{\kappa^{2}+\lambda_{D}}\right) \lambda_{D}+\frac{\beta}{1-\beta} \kappa^{2} \lambda_{D}\left(y^{*}\right)^{2} \frac{\lambda_{D}+\kappa^{2}}{\left(\kappa^{2}+\lambda_{D}(1-\beta)\right)^{2}} .
$$

In particular, in the case of $\lambda_{D}=0$, condition (4) boils down to:

$$
\sum_{t}^{\infty} \beta^{k-t}\left(\pi_{t}^{2}\right) \leq 0, \text { for every period t. }
$$

As a result, when $\lambda_{D}=0, \pi_{t}=0$ for any period after the trap. In particular, this holds whatever the value of the discount factor $\beta$.

This leads to the following proposition:

Proposition 1 (Full information). When $\gamma>0$, the Ramsey allocation is sustainable by $a \lambda_{D^{-}}$ central banker, only if $\lambda_{D}$, the weight on the output-gap stabilization objective, is intermediate, i.e. $\lambda_{D} \in\left[\underline{\lambda}_{D}, \bar{\lambda}_{D}\right]$ where $0<\underline{\lambda}_{D} \leq \bar{\lambda}_{D}<\infty$. In this case, inflation is fully stabilized before the trap.

In addition, there exists at least one central banker that triggers no inflation before the trap when $\lambda_{D}$ is lower than $\underline{\lambda}_{D}$, that is the conservative central banker $\left(\lambda_{D}=0\right)$.

\footnotetext{
${ }^{6}$ For a given level of $\gamma$, there also exists a specific value of $y^{*}$ such that $\pi_{t}=0$ as $y_{t}=y^{*}$, but we discard such solution as we concentrate on cases where the discretionary central banker stabilizes inflation for all values of $\gamma$.
} 
Proof. See Appendix B.1

Proposition 1 provides several insights. First of all, inflation stabilization before a trap occurs can be achieved both by credible - with intermediate $\lambda_{D}$ - and conservative $-\lambda_{D}=0$ - central bankers. Second, only the first category of central bankers is able to sustain the Ramsey allocation and especially to implement forward guidance policy. For low weight on the output-gap stabilization objective, the central banker is not able to sustain this allocation. In particular, the conservative central banker will never credibly implement any kind of forward guidance policies after the liquidity trap as he can achieve full inflation stabilization afterward.

The presence of the zero lower bound is key to obtain this disconnection between inflation stabilization before the trap and ability to sustain forward guidance. In the absence of ZLB $(\gamma=0)$, the Ramsey allocation coincides with the preferred outcome of the conservative central banker $\left(\lambda_{D}=0\right)$. Yet, such coincidence that motivated Rogoff (1985)'s delegation result, does not arise anymore when liquidity trap requires to implement forward guidance policy.

In the end, under perfect information, positive inflation bias $\left(\lambda_{D}>0\right)$ is required to ensure both optimal policies before and after the trap.

In the rest of the paper, we make the following assumption:

Assumption 3. The benevolent central banker is able to achieve the Ramsey allocation, i.e. $\lambda \in\left[\underline{\lambda}_{D}, \bar{\lambda}_{D}\right]$.

This assumption discards situations in which the social welfare does not allow reputation concerns to sustain the Ramsey allocation.

\section{Model under imperfect information}

We now turn to the equilibrium under imperfect information on central bankers' types. First, we define such an equilibrium, and second we characterize it. In this section and in Section 4 , we focus on the case where the central banker's types are either benevolent $\left(\lambda_{D}=\lambda\right)$ or conservative $\left(\lambda_{D}=0\right)$. We consider this case as our benchmark case, as we have shown in proposition 1 that these two types stabilize inflation prior to a liquidity trap but the former is credible to commit to forward guidance policy while the latter is not. To phrase it differently, we are interested in the implementation of the third-best policy, that is the best policy under the ZLB constraint and imperfect information on central banker's type. 


\subsection{Beliefs and equilibrium definition.}

We denote agents beliefs about the central banker's type in period t by $q_{t} \in[0,1]$, with the following meaning: when $q_{t}=1$, agents are sure that the central banker is benevolent, while, when $q_{t}=0$, agents are certain that the central banker is conservative.

In particular, $q_{-\infty}$ denotes the agents' prior beliefs. For simplicity, we drop the index: $q_{-\infty}=q$.

Definition 1. An equilibrium is an allocation $\left\{\pi_{t}, y_{t}\right\}_{t}$, a sequence of policy decisions $\left\{i_{t}\right\}_{t} a$ sequence of beliefs $\left\{q_{t}\right\}_{t}$ such that, for each period $t$ :

- Given the sequences of beliefs and policy decisions, $\pi_{t}$ and $y_{t}$ solve (NKPC) and (EE), where expectations are consistent with beliefs and policy.

- Given sequences of beliefs and allocations, both central bankers optimize their objective function under the constraint (ZLB).

- Private agents update beliefs on the central banker's type following the Bayes' law whenever possible.

Such a definition of equilibrium allows for trigger strategies and sustainability based on reputation but also asymmetric information on the central banker's preference. In the following, we suppose that economic agents always coordinate on the best sustainable equilibrium.

\subsection{Central banker's actions and payoffs.}

After the trap. To start with, let us note that once the trap ends, the conservative central banker has no incentive to imitate the benevolent central banker anymore as he can fully stabilize inflation. This implies that our problem will behave in the long run as in the full information case. This helps us to simplify the dynamic component of our signaling game.

Separating before and during the trap. Let us first describe the benevolent central banker's policy when separating between types occurs. Such a separating emerges if and only if the benevolent central banker's policy implements a policy that prevents the conservative central banker from imitating his policy prior to the liquidity trap. Thus, it has to exist a date $t$ strictly lower than the first period of the liquidity trap, denoted by $t_{l}$, such that the following incentive compatibility constraint is satisfied:

$$
\sum_{\tau=t}^{\infty}(\beta(1-\gamma))^{\tau-t}\left[\frac{1}{2} \pi_{\tau}^{2}+\beta \gamma L_{t_{l}}^{0}(S, 1)\right] \geq \sum_{\tau=t}^{\infty}(\beta(1-\gamma))^{\tau-t} \beta \gamma L_{t_{l}}^{0}(S, 0),
$$


where we denote by $L_{t_{l}}^{0}(S, 0)$ the loss of the conservative central banker during and after the trap when he follows his own policy and by $L_{t_{l}}^{0}(S, 1)$ when he replicates the benevolent central banker's policy before and during the trap. As the liquidity trap can occur at any moment $\left(t_{l}\right.$ can be any date), the incentive constraint REV has to be true at the time of the optimization which is supposed to be equal to $-\infty .^{7}$ Therefore, the resulting problem for the benevolent central banker in a separating equilibrium and assuming commitment ability writes:

Problem 3 (Benevolent central banker's program under separating equilibrium). Given the following sequence of beliefs: $q_{t}=1$ for all periods,

$$
\min _{\left\{i_{t}\right\}_{t}} L_{-\infty}
$$

under the incentive compatibility constraint (REV) at date $t=-\infty$ and constraints (NKPC), (EE) and (ZLB) at each date.

The following proposition describes the solution of the benevolent central banker's problem under separating equilibrium.

Proposition 2. The solution of Problem 3 takes the following form:

(i) for any period $t$ before the trap, inflation is constant $\left(\pi_{t}=\pi\right)$ and solves:

$$
\pi^{2} \geq \beta \gamma\left[L_{t_{l}}^{0}(S, 0)-L_{t_{l}}^{0}(S, 1)\right]
$$

(ii) During and after the trap, inflation $\pi_{t}$ is lower than the perfect information solution.

Furthermore, under Assumption 3, this solution is sustainable by the benevolent central banker.

Proof. See Appendix B.2.

First, the optimal policy in a separating equilibrium is to set a positive and constant level of inflation prior to the liquidity trap. This positive level ensures truth-telling of the conservative central banker. Second, the benevolent central banker also implements a less powerful forward guidance policy to disincentive the conservative central banker to imitate him. Finally, while this optimal policy differs from the Ramsey allocation in perfect information, it remains sustainable. The benevolent central banker does not lower inflation before the trap to ensure the incentive compatibility constraint and after the trap the incentive to deviate decreased as he implements a weaker forward guidance.

\footnotetext{
${ }^{7}$ Because the incentive compatibility constraint holds for $t=-\infty$, the equilibrium is stationary and does not depend on an arbitrary date of optimization.
} 
Pooling before and during the trap. We now turn to the description of the benevolent central banker's policy when both central bankers are pooled. It is worth noting that because the problem before the liquidity trap is stationary, the sequence of beliefs in pooling verifies: $q_{t}=q$ for periods t before and during the trap, and $q_{t}=1$ for periods after the trap.

Problem 4 (Benevolent central banker's program under pooling.). Given the sequence of beliefs $q_{t}=q$ for $t \leq t_{l}$ and $q_{t}=1$ after the trap,

$$
\min _{i_{t}, t} L_{-\infty}
$$

under the constraints (NKPC), (EE) and (ZLB).

Let us now describe how private beliefs $q$ affect central banks' outcomes once the economy is in a liquidity trap. Real functions $L_{t_{l}}(P, q)$ and $L_{t_{l}}^{0}(P, q)$ denote the losses of the benevolent and the conservative central bankers once the liquidity trap hits the economy given private beliefs on the central banker's type, $q$.

Proposition 3. The solution to Problem 4 is to implement zero inflation prior to the liquidity trap, that is $\forall t<t_{l}, \pi_{t}=0$. Furthermore, central bankers' losses satisfy:

(i) The welfare loss during the liquidity trap $L_{t_{l}}(P, q)$ is decreasing in $q$. In addition, $L_{t_{l}}(P, 1) \leq$ $L_{t_{l}}(S, 1)$, where $L_{t_{l}}(S, 1)$ denotes the loss in the signaling case.

(ii) The conservative central banker's loss $L_{t_{l}}^{0}(P, q)$ is decreasing in $q$.

Proof. See Appendix B.3.

The more agents are convinced that the central banker is benevolent ( $q$ approaching 1 ), the lower the losses of both central bankers due to the liquidity trap. Indeed, larger beliefs mean that more agents are convinced that the central banker can commit to forward guidance policy which is beneficial for both types. Even though conservative central banker will, ex post, deviate from forward guidance commitment, he benefits from the private beliefs in forward guidance's future easing monetary policy. In the end, both central bankers have an incentive to raise beliefs $q$.

Note that Proposition 3 remains silent about the sustainability by the benevolent central banker of the solution to Problem 4. When beliefs are such that $q<1$, this may require the central banker to engage into more monetary stimulus after the end of the trap, so as to compensate for lack of credibility. ${ }^{8}$ In turn, this can make the solution to Problem 4 not

\footnotetext{
${ }^{8}$ See Andrade et al. (2015) for a similar insight on the hump-shaped forward guidance with respect to private beliefs on commitment ability.
} 
sustainable, in particular, after the trap and the benevolent central banker's best policy is such that his loss after the trap may be lower than the loss obtained under commitment. To keep tractability, we make the following assumption in the remaining of the section:

Assumption 4. The solution to Problem 4 is sustainable by the benevolent central banker.

Such an assumption works against us as the sustainability of the solution to Problem 4 actually increases the value of being pooled for the benevolent central banker and so reduces the need for signaling. Yet, it does not affect the incentive constraint of the conservative central banker, that determines the level of signaling inflation, as it will become clear in the next paragraph.

Summary. We have successively examined separating and pooling cases. Separating will be eventually chosen if separating dominates pooling from the benevolent central banker point of view. Thus, separating emerges if and only if the following two incentive constraints hold:

$$
\begin{aligned}
& \beta \gamma\left(L_{t_{l}}(P, q)-L_{t_{l}}(S, 1)\right) \geq \pi^{2}+\lambda\left[\left(y-y^{*}\right)^{2}-\left(y_{p}-y^{*}\right)^{2}\right] \\
& \pi^{2} \geq \beta \gamma\left[L_{t_{l}}^{0}(S, 0)-L_{t_{l}}^{0}(S, 1)\right]
\end{aligned}
$$

with $\pi$ and $y$ are inflation and the output gap chosen by the benevolent central banker before the trap in case of separating equilibrium. In the pooling equilibrium, inflation is zero but the output gap $y_{p}$ is positive to compensate deflationary expectations due to future zero lower bound.

Equation (7) captures the incentive constraint for the benevolent central banker not to be pooled with the conservative central banker and who then enjoys the full gains from forward guidance, i.e. $\beta \gamma\left(L_{t_{l}}(P, q)-L_{t_{l}}(S, 1)\right)$. Equation (8) is the incentive constraint for the conservative central banker not to mimic the benevolent central banker's policy and who suffers from loss before the trap. In the end, the inflation rate before the trap should be sufficiently low not to deter too much the benevolent central banker's outcome, but it should be sufficiently high to cause losses to the conservative central banker to convince him not to mimic the benevolent central banker's policy.

\subsection{Equilibrium description.}

The following theorem describes the equilibrium outcome of the signaling game played by the two central bankers. In particular, it identifies when agents are able to elicit information on central bankers' type, both before and after the trap. 
Theorem 4. When the inflation bias $y^{*}$ is sufficiently low, there exists $\bar{q}<1$ such that the equilibrium is as follows:

(i) For any period $t$ after the trap, the equilibrium is separating and agents are certain about the central banker's type: $q_{t} \in\{0,1\}$.

(ii) For any period $t$ before and during the trap:

- If $q \leq \bar{q}$, the equilibrium is separating $\left(q_{t} \in\{0,1\}\right)$ and the inflation rate set by the benevolent central banker is positive before the trap (strictly positive when $q>0$ ).

- Otherwise, the equilibrium is pooling $\left(q_{t}=q\right)$ and the inflation rate equals 0 for both central banker types before the trap.

Proof. See Appendix.

When prior beliefs are too biased towards the conservative central banker $(q \leq \bar{q})$, the cost of imperfect credibility on the effectiveness of the forward guidance by the benevolent central banker is so great that the central banker increases inflation before the trap as a costly signaling device. This results in a separating equilibrium.

When prior beliefs are biased towards the benevolent central banker, such costly signaling is not desirable any more, as the resulting gains in the trap are lower. This results into a pooling equilibrium. At the limit, when economic agents fully believe that the central banker is benevolent, there is no reason to avoid pooling as pooling does not limit the efficiency of the forward guidance while the incentive compatibility constraint (REV) imposes a suboptimal constraint to the optimization program. That explains why the threshold, $\bar{q}$ is strictly lower than one.

In either case, agents observe perfectly the type of central banker after the trap, except in the degenerate case where $q=0$, i.e. when the prior belief that the central banker is benevolent equals 0 .

The existence of separating equilibria. The next proposition states necessary conditions to obtain separating equilibrium and determines how the set of such separating equilibria evolves with respect to the inflation bias.

Proposition 5. $\bar{q}>0$ only if $\lambda>0$. In addition, $\bar{q}$ is strictly increasing in $y^{*}$, when $\lambda>0$ and increasing with $\lambda$.

Proof. See Appendix B.5. 
Proposition 5 clarifies the condition under which the benevolent central banker signals his type (separating equilibrium). The mechanism is as follows. By raising inflation before the trap the benevolent central banker incurs a loss due to the quadratic term in inflation in its loss similar to the conservative type; but he also benefits from lower output loss because the output gap is closer to the efficient level, $y^{*}$. This latter gain is specific to the benevolent central banker, crucially depends on the weight on output-gap stabilization in the welfare function and explains why he enjoys fewer costs compared to the conservative type when raising inflation.

Inflation, beliefs and probability of ZLB. To minimize the required inflation rate, equation (8) holds with equality. This leads to the following corollary:

Corollary 6. Before the trap, the inflation rate in the separating equilibrium (when $q=1$ ), $\pi$, weakly increases with:

(i) The probability of a liquidity trap $\gamma$ and there is no inflation when $\gamma=0$. When $q \leq \bar{q}$, $\pi$ strictly increases with $\gamma$.

(ii) The intensity of the liquidity trap $r$. When $q \leq \bar{q}$, $\pi$ strictly increases with $r$.

Proof. See Appendix.

More likely and more intense liquidity traps imply a higher inflation target, as the gains for the conservative central banker type from mimicking the benevolent central banker are higher and the loss for this latter type from pooling is higher.

Do more frequent liquidity traps imply more frequent signaling? How does the threshold $\bar{q}$ evolve with respect to the probability of liquidity traps? This is determined by incentive constraints (7) and (8) and, more specifically, how the term:

$$
\left[\left(y-y^{*}\right)^{2}-\left(y_{p}-y^{*}\right)^{2}\right]=\left(y-y_{p}\right)\left(y+y_{p}-2 y^{*}\right)
$$

evolve with respect to $\gamma$.

Increasing inflation involves a direct welfare loss but also affects welfare through the higher level of the output gap. On the other hand, in a separating equilibrium, the benevolent central bank is better able to smooth inflation and the output gap during the liquidity trap. In turn, lower expected deflationary pressures reduce monetary accommodation before the trap (that allows to maintain inflation at 0) and, hence, the level of output. In the end, in a separating equilibrium, the sign of output in comparison with a pooling one is ambiguous. 
We however report the variation of the threshold $\bar{q}$ with respect to the probability $\gamma$, when this probability is sufficiently small:

Proposition 7. For sufficiently small probability of $Z L B \gamma, \bar{q}$ is increasing in $\gamma$.

Proof. See Appendix B.7.

As a result of this proposition, the level of inflation can even be a discontinuous function of the probability of ZLB. Let $q$ be above but arbitrarily close to $\bar{q}(\gamma)$. The inflation rate is then zero before the trap. A small increase in the probability of hitting the ZLB of $d \gamma$ leads $q$ to be below $\bar{q}(\gamma+d \gamma)$ and so to a strictly positive inflation rate.

\subsection{Time-varying probabilities of ZLB.}

Suppose that before a trap occurs, there are two states associated with two different probabilities of ZLB. For simplicity, we assume that the natural rate of interest is the same in these two states. The corresponding transition matrix is:

$$
\left(\begin{array}{ccc}
\Pi_{1} & 1-\Pi_{1}-\gamma_{1} & \gamma_{1} \\
1-\Pi_{2}-\gamma_{2} & \pi_{2} & \gamma_{2} \\
0 & 0 & 1
\end{array}\right)
$$

with $\gamma_{1}>\gamma_{2}$, that is the ZLB is more likely in state 1 than in state 2 .

Proposition 8. A necessary condition for the equilibrium to be separating is that level of inflation in state $i$ implemented by the benevolent central banker satisfies the following condition:

$$
\pi_{i}^{2}=\beta \gamma_{i}\left(L_{t_{l}}^{0}(S, 0)-L_{t_{l}}^{0}(S, 1)\right)
$$

Proposition 8 implies that the higher the probability of hitting the ZLB, the higher the level of signaling inflation. Furthermore, the signaling level of inflation only depends on the current probability to reach the zero lower bound and, in particular, the signaling level of inflation $\left(\pi_{1}\right.$ and $\left.\pi_{2}\right)$ is independent of transition probabilities from state to state outside the liquidity trap $\left(\Pi_{1}\right.$ and $\left.\Pi_{2}\right)$.

To gain intuition from this result, let us recall that, because there is always a possibility to stay in the same state before reaching the liquidity trap, equation (9) needs to be satisfied in both states. Conversely, if equation (9) holds for both states, any linear combinations of those inequalities are also satisfied ensuring separating equilibrium whatever the states history. 
Remark. Note that under the condition of Proposition 8, the equilibrium is fully separating, i.e. separating in all states before the trap and whatever the history of state realisation. More complex situations can possibly arise where the equilibrium is pooling in some state before the trap but separating in others. In such situations, the signaling inflation rate is higher in separating states than in the case where the benevolent central banker signals himself in all states and pooling states are pooling only if any previous state was pooling as well.

\section{How large is signaling inflation?}

In this section, we quantitatively assess how great the level of inflation to signal the central banker's type to private agents should be.

\begin{tabular}{lcc}
\hline \hline Definition & Parameters & Value \\
\hline \multicolumn{3}{c}{ Structural parameters } \\
Discount factor & $\beta$ & 0.99 \\
Risk aversion & $\sigma$ & 1 \\
NKPC slope & $\kappa$ & 0.02 \\
Interest rate steady state & $\bar{i}$ & 0.01 \\
\multicolumn{2}{c}{ Liquidity trap parameters } & \\
Probability of ZLB & $\gamma$ & 0.007 \\
Size of the shock & $r$ & 0.015 \\
Duration of the trap & $N_{\text {trap }}$ & 12 \\
\multicolumn{4}{c}{ Social welfare parameters } \\
Weight of the output gap & $\lambda$ & 0.025 \\
Inflation bias & $y^{*}$ & 0.0625 \\
\hline \hline
\end{tabular}

Table 1 - Baseline calibration

In Table 1, we report our baseline calibration. Most of structural parameters are standard and calibrated following Woodford (2003) or Eggertsson and Woodford (2003). We calibrate the frequency of the liquidity trap to a very low level of $0.007 \%$ per quarter as in Coibion et al. (2012). The size and the duration of the trap are consistent with the estimates of Barsky et al. (2014) for the post world war II US economy. The trap is hence rare but when occurring severe and relatively long. Finally, concerning the social welfare parameters, there is no clear consensus in the literature about the weight of the output-gap, so we choose to calibrate this parameter to an intermediate value of 0.025 . Finally, we calibrate the inflation bias, $y^{*}$ 
to 0.0625 consistently with Woodford (2003). We provide for many competing calibrations below.

Optimal signaling inflation. Table 2 reports the level of optimal signaling inflation and the threshold for prior beliefs above which pooling emerges, $\bar{q}$.

In our baseline specification the inflation level is slightly lower than 2 while the threshold belief, $\bar{q}$ is around 0.3. This suggests that the inflation level is significantly positive even if below the often chosen inflation target of $2 \%$. However, signaling commitment ability is only required if prior beliefs are very biased toward the conservative central banker. In Appendix A, Figure 1 displays inflation, the output gap and the nominal interest rate when the economy is hit by a liquidity trap during 12 periods for different prior beliefs in the baseline calibration.

\begin{tabular}{ccc}
\hline \hline Cases & Inflation level, $\pi$ & threshold beliefs, $\bar{q}$ \\
\hline baseline & 1.5 & 0.30 \\
& Trap spell & \\
Shorter $\left(N_{T R A P}=8\right)$ & 0.5 & 0.10 \\
Longer $\left(N_{T R A P}=16\right)$ & 3.4 & 0.14
\end{tabular}

Frequency of the ZLB

Less frequent $(\gamma=0.005) \quad 1.3 \quad 0.31$

Slightly more frequent $(\gamma=0.02) \quad 2.5 \quad 0.30$

More frequent $(\gamma=0.05) \quad 4.0 \quad 0.32$

Severity of the ZLB

$\begin{array}{lll}\text { Less severe }(r=0.04 \text { annualized }) & 1.0 & 0.33\end{array}$

More severe $(r=0.08$ annualized $) \quad 2.0 \quad 0.29$

Risk aversion
Low risk aversion $(\sigma=1.5)$
0.9
0.21
High risk aversion $(\sigma=1 / 2)$
3.7
0.16

Output-gap weight

$\begin{array}{ccc}\text { Small weight }(\lambda=0.0025) & 1.5 & 0.06 \\ \text { Large weight }(\lambda=0.05) & 1.5 & 1\end{array}$

Inflation bias

Close to zero $\left(y^{*}=0.00625\right) \quad 1.5 \quad 0.27$

Large bias $\left(y^{*}=0.625\right) \quad 1.5 \quad 1$

Table 2 - Optimal signaling inflation 
Probability of the ZLB is a key determinant of the inflation level. For instance, a probability of $5 \%$ per quarter leads to more than $4 \%$ inflation. Equation (8) proves that the signaling level of inflation is proportional to the root square of the probability of ZLB:

$$
\pi \geq \sqrt{\beta \gamma\left[L_{t_{l}}^{0}(S, 0)-L_{t_{l}}^{0}(S, 1)\right]}
$$

and, so, the marginal effect of a change in probability when the probability of hitting the ZLB is close to 0 is large.

Besides, the threshold $\bar{q}$ below which separating equilibria arises is very sensitive to both the output gap weight in the central banker's objective function $\lambda$ and the inflation bias $y^{*}$. In particular, if one of these two preference parameters are sufficiently large, then separating equilibrium emerges whatever the beliefs. Conversely, the signaling level of inflation decreases only modestly with this parameter. This is consistent with the fact that these parameters determine how much the benevolent central banker's preferences are different from the conservative one while they do not enter into the latter one's preferences.

Risk aversion is also crucial as it directly affects the effectiveness of forward guidance. The higher the risk aversion, the lower the inflation, as the gain from forward guidance is lower. Inflation goes from $0.9 \%$ to $3.7 \%$ for sigma between 1.5 to 0.5 . Given the uncertainty and the importance of this parameter, the choice of a standard logarithmic utility function $(\sigma=1)$ appears a neutral stance.

Signaling inflation and inflation target. We have assumed that the optimal steady state level of inflation is zero in the absence of inflation bias. However, there may be reasons to target a positive level of inflation in the welfare function. The European Central Bank, for instance, invoks three reasons to target a positive rather than a zero level of inflation: limit the probability of a liquidity trap, take into account possible positive bias in the measurement of price level changes and a sufficient buffer to avoid deflation in one country in the euro area due to inflation differentials. These last two reasons are beyond the scope of this paper. We hence wonder whether our results would be affected by such a positive price stability definition. To answer this question, we modify the preferences of central bankers by adding a positive inflation target, $\bar{\pi}$ as follows: ${ }^{9}$

$$
\tilde{L}_{t}^{\lambda_{D}}=\frac{1}{2} E_{s} \sum_{t=s}^{\infty} \beta^{t-s}\left[\left(\pi_{t}-\bar{\pi}\right)^{2}+\lambda_{D}\left(y_{t}-y^{*}\right)^{2}\right]
$$

\footnotetext{
${ }^{9}$ We do not consider cases in which one of the central bankers targets a positive level and not the other as this would generate de facto a strong incentives not to imitate the other central banker.
} 
We, then, can rewrite the incentive constraints with a positive inflation target and compute the threshold $\bar{q}$ and the level of inflation, $\pi$. Assuming that the inflation target $\bar{\pi}$ is $2 \%$ in annual term, we find the same level of threshold, $\bar{q}=0.3$ and a level of inflation needed to signal the benevolent central banker type around $3.4 \%$ in our benchmark calibration. As a result, our main result does not change much when including a positive inflation target.

It is worth noticing that in this exercise we do not take into account the impact of the inflation target on the probability of the liquidity trap as the shock is sufficiently large to always generate a zero lower bound once it hits the economy. However, the probability of ZLB we consider is consistent with historical data for the US economy that has experienced positive average inflation.

Discounted Euler equation. The level of signaling inflation depends on the gains of forward guidance at the zero lower bound. Yet, as argued by Del Negro et al. (2013) forward guidance may be less effective compared to what is predicted by the model. ${ }^{10}$ In the following, we integrate a discounted Euler equation as introduced by McKay et al. (2015) to account for this lower efficiency: ${ }^{11}$

$$
y_{t}=\alpha E_{t} y_{t+1}-\frac{\zeta}{\sigma}\left(i_{t}-E_{t} \pi_{t+1}-r_{t}^{n}\right)
$$

where both $\alpha$ and $\zeta$ parameters are below 1 and hence reduce both the severity of a natural rate of interest shock and forward guidance effectiveness.

For comparability reasons, we calibrate all parameters as in the baseline calibration of McKay et al. (2015). The risk aversion is set to $\sigma=2$, duration of the trap is set to 20, the size of the shock is $-2 \%$ in annual term. The discounting parameters are calibrated as in the benchmark case of McKay et al. (2015): $\alpha=0.97$ and $\zeta=0.75$.

In this case, for a probability of ZLB as in Coibion et al. (2012): inflation is 0.5. This result is not due to limited effectiveness of forward guidance but rather due to the small recession and hence the small loss generated by the shock they consider, as well as the limited impact due to the discounted Euler equation and the high risk aversion. Indeed, in the discretionary case output falls by $5 \%$ and inflation by less than $4 \%$.

We thus can consider this calibration as a very conservative one that gives a lower bound for the signaling inflation level. Even if this level is rather small, it does not mean that it is

\footnotetext{
${ }^{10}$ Note that an intermediate level of q can also explain lack of effect of forward guidance in our model. See Andrade et al. (2015) for the connection between private beliefs and the so-called forward guidance puzzle.

${ }^{11}$ They actually show that incomplete markets and heterogeneous agents can reduce the efficiency of forward guidance and they then show that the discounted Euler equation is a shortcut to reproduce their findings in a representative agents formulation.
} 
always negligible. When the probability of ZLB is high (5\% per quarter) and the severity of the crisis is more in line with estimated values (Barsky et al., 2014), inflation signaling can become significantly non zero (4\% in this particular case). This suggests that even if the exact level of inflation needed to signal commitment ability may change from one specification to the other, the level of inflation can easily be greater than two when the liquidity trap becomes more likely and/or more severe.

\section{Inflation-biased central banker.}

Raising inflation to signal commitment ability may also be costly as private agents may mistakenly believe that the central banker acts under discretion with an inflation bias. In this section, we investigate the incentives of benevolent central banker to raise inflation when such an inflation can also be taken as a sign of lack of commitment.

To this purpose, we assume that central bankers may also differ with respect to their discount factor. To simplify our problem, we assume that the discount factor can take two values: a high one as in the benchmark model, $\beta$ and a low one equals to 0 for simplicity. We call this latter type, the inflation-biased central banker. This latter type cannot sustain the Ramsey equilibrium whatever the weight on output-gap stabilization, $\lambda_{D}$.

We first show that the benevolent central banker can be optimally pooled with the inflation-biased central banker. Then, we show that the benevolent central banker's incentive to be pooled with the inflation-biased central banker ex ante can be strengthened by the former's ability to dis-inflate the economy once the liquidity trap ends.

The model. On top of the two types that we already considered, we now consider that central bankers' preferences can also be $\beta=0$ and $\left.\left.\lambda_{D} \in\right] 0, \infty\right) .{ }^{12}$ We thus now denote by a bi-dimensional vector the central banker's type, $\left(\beta, \lambda_{D}\right)$. The prior beliefs that the central banker is benevolent, $(\beta, \lambda)$, is denoted by $q$, the one that he is conservative, $(\beta, 0)$, by $q_{\text {cons }}$ and there is a probability distribution function $g$ so that the prior belief that central banker is of type $\left(0, \lambda_{D}\right)$ is $g\left(\lambda_{D}\right)$. Prior beliefs have to sum to one so, $q+q_{\text {cons }}+\int_{0}^{\infty} g\left(\lambda_{D}\right) d \lambda_{D}=1$.

Note that the central banker with a positive $\lambda_{D}$ but with a zero discount factor $(\beta=0)$ cannot act strategically and so, it would be easy for the benevolent central banker to always separate himself from the inflation-biased central banker, just by creating noise in the ex ante rate of inflation. Yet, such strategy is not robust to positive discount factors for the inflationbiased central banker and, in order not to lose generality, we impose that the benevolent

\footnotetext{
${ }^{12}$ We exclude 0 because it would be redundant with the conservative central banker.
} 
central banker adopts a constant rate of inflation before a trap occurs.

\subsection{Incentive constraints.}

Positive inflation prior to the liquidity trap. As long as the benevolent central banker implements positive inflation, $\pi$, prior to the liquidity trap, there always exists an inflationbiased central banker leading to the same level of inflation. The benevolent central banker is hence pooled with a discretionary central banker $\left(0, \lambda_{D}^{*}\right)$ :

$$
\lambda_{D}^{*}=\kappa \frac{y^{*}-\pi \kappa}{\left(\pi(1-\beta(1-\gamma))-\beta \gamma \pi_{l}\right)}
$$

where $\pi_{l}$ is the level of inflation during the trap.

As a result, before the trap, beliefs over central bankers with no discount factor go to zero except the one corresponding with $\lambda_{D}^{*}$. The equilibrium is never fully separating but only partially. However, the exact reallocation of beliefs is indeterminate and many equilibria are compatible with our Bayesian Perfect Equilibrium definition. We thus parametrize beliefs once such a partial pooling emerges. We denote by $q^{\prime}$ and $1-q^{\prime}$ the beliefs associated with the benevolent and the $\left(0, \lambda_{D}^{*}\right)$-type central bankers. ${ }^{13}$

After the trap, by choosing a different level of inflation, the benevolent central banker can distinguish himself from the $\left(0, \lambda_{D}^{*}\right)$ central banker type so that the equilibrium becomes fully separating as soon as the trap is over.

Zero inflation case. When the benevolent central banker sets a zero inflation rate prior to the trap, economic agents hence form (indeterminate) beliefs over only two central banker's types: either the benevolent type, $q^{\prime \prime}$, or the conservative's one, $1-q^{\prime \prime}$. Indeed, we exclude that an inflation-biased central banker attributes zero weight on the output gap stabilization objective.

Incentive constraint of the benevolent central banker. The benevolent central banker prefers setting a positive inflation level prior to the liquidity trap if the gain in terms of forward guidance, $L_{t_{l}}\left(0, q^{\prime \prime}\right)-L_{t_{l}}\left(\pi, q^{\prime}\right)$, compensates the loss due to positive inflation prior to the trap, hence:

$$
\beta \gamma\left(L_{t_{l}}\left(0, q^{\prime \prime}\right)-L_{t_{l}}\left(\pi, q^{\prime}\right)\right) \geq \pi^{2}+\lambda\left[\left(y\left(0, q^{\prime \prime}\right)-y^{*}\right)^{2}-\left(y\left(\pi, q^{\prime}\right)-y^{*}\right)^{2}\right],
$$

\footnotetext{
${ }^{13}$ Because of the Bayes' law, beliefs satisfy: $1-q \geq 1-q^{\prime} \geq g\left(\lambda_{D}^{*}\right)$ and $q^{\prime} \geq q$.
} 
where $y\left(0, q^{\prime \prime}\right)$ and $y\left(\pi, q^{\prime}\right)$ are the output gap if the benevolent central banker is pooled with the conservative and the inflation-biased central bankers respectively. A rise in inflation, $\pi$ trigger two effects. First, it increases the loss before the liquidity trap through higher inflation. Second, it affects expectations at the liquidity trap through a change in $\lambda_{D}^{*}$. This second effect can potentially reduce or enhance the severity of the deflation during the trap.

Incentive constraint of the conservative central banker. The conservative central banker imitates the benevolent central banker if the gains from forward guidance dominates the costs of inflation prior to the liquidity trap. Therefore, the relevant incentive constraint is:

$$
\pi^{2} \geq \beta \gamma\left(L_{t_{l}}^{0}\left(\pi, q^{\prime}\right)-L_{t_{l}}^{0}\left(0, q^{\prime}\right)\right)
$$

Contrary to Section 3, the loss of the conservative if mimicking the benevolent central banker, $L_{t_{l}}^{0}\left(\pi, q^{\prime}\right)$, depends on the inflation level $\pi$ chosen by the benevolent central banker. Indeed, the third central banker type characterized by $\left(0, \lambda_{D}^{*}\right)$ depends on the inflation level $\pi$ and he matters for the efficiency of the forward guidance. For instance, if $\pi$ is low, the benevolent central banker will be pooled with a low $\lambda_{D}^{*}$ type producing low level of inflation after the liquidity trap making the liquidity trap more costly, on the contrary high level of $\pi$ and hence high level of $\lambda_{D}^{*}$ will reduce the cost of the trap. In other words, $L_{t_{l}}^{0}\left(\pi, q^{\prime}\right)$ is decreasing with $\pi$ for a given $q^{\prime}$. Finally, if economic agents do not consider inflation-biased central banker in their beliefs, $q^{\prime}=1$, then inequalities (12) and (8) are identical.

\subsection{Equilibrium description}

Proposition 9. The equilibrium is as follows

(i) For any period $t$ after the trap, the equilibrium is separating and agents are certain about the central banker's type.

(ii) For any period $t$ before and during the trap:

- If $q^{\prime \prime} \leq \bar{q}$, the equilibrium is partially separating: the benevolent central banker is pooled with the $\left(0, \lambda_{D}^{*}\right)$ central banker but separated from the conservative one. The inflation rate set by the benevolent central banker is positive before the trap.

- Otherwise, the benevolent central banker is pooled with the conservative central banker and the inflation rate equals 0 before the trap. 
The introduction of central bankers with zero discount factor does not qualitatively modify results from Theorem 4 . The benevolent central banker desires not to be mistakenly considered as conservative if prior beliefs are strongly biased toward this former type. In this case, inflation acts as a costly signal for the benevolent central banker.

Connection with Coibion et al. (2012). In some equilibria, learning due to signaling leads to a degenerate distribution peaked on the inflation-biased central banker, i.e. $q_{\lambda_{D}^{*}}=1$. In this case, signaling is impotent to reveal the commitment ability to agents that attribute inflation only to inflation bias. In the end, ex ante, such a situation corresponds to the optimal inflation target when the central banker acts under discretion as in Coibion et al. (2012). They show that the buffer stock effect of higher inflation rate already explain an inflation target of at least $1.5 \%$ in their benchmark case.

Inflation-biased central bankers with non-zero discount factor. A positive discount factor of inflation-biased central bankers might reduce the ex ante incentive of the benevolent central banker to be pooled with them. Indeed the ex-post cost of disinflation policy is higher because inflation-biased central bankers will choose to strategically mimic the benevolent central banker's policy after the trap. Finally, the disinflation will require higher real rates leading to larger fall of the output-gap. However, in the long run, as long as $\beta$ is sufficiently low, separating will emerge. ${ }^{14}$

\section{Cost-push shocks before the trap.}

In this section, we assess the robustness of our analysis to the presence of cost-push shocks before de trap. As the monetary policy response to such shocks cannot ensure perfect inflation and output stabilization, the corresponding optimal response also requires commitment ability. ${ }^{15}$ In the end, such shocks may potentially trigger separation so that signaling through higher inflation is no longer necessary. Our main result is that higher inflation is still required to ensure separation, if the shocks before the trap remain sufficiently small. Indeed, when experiencing small shocks, the conservative central banker has still an incentive to mimic the benevolent central banker, anticipating larger gains associated with the liquidity trap in the future.

\footnotetext{
${ }^{14}$ Otherwise, it means that the discount factor is sufficiently large to make the Ramsey allocation sustainable and inflation-biased central banker does not differ from the benevolent central banker anymore.

${ }^{15}$ Alternatively, this can be additional liquidity trap shocks.
} 
Let us suppose that cost-push shocks, $\sigma u_{t}$ can hit the economy before the liquidity trap. These shocks modify the (NKPC) as follows:

$$
\pi_{t}=\beta E_{t} \pi_{t+1}+\kappa y_{t}+\sigma u_{t}
$$

In addition, we assume that these shocks are bounded, $u_{t}<M<\infty$, and are sufficiently small, $\sigma<<1$.

First, let remark that it is possible to derive all trajectories (under separating and pooling) as perturbed trajectories around trajectories described in Appendix A. Let us explore how the presence of cost-push shocks affects central bankers' incentive constraints.

The conservative central banker. Prior to the trap, the conservative central banker can achieve full stabilization of inflation, and hence no loss. His only loss thus results from the liquidity trap, $L_{t_{l}}^{0}(S, 0)$. However, when the conservative central banker replicates the benevolent central banker's policy, the loss is history dependent and depends on Lagrange multiplier $\tilde{\mu}$ :

$$
\mathcal{L}_{t}=\pi_{s}^{2}+\beta E_{s} \sum_{t>s} \beta^{t-s}\left[(1-\gamma) \pi_{t}^{2}+\gamma L_{t_{l}}^{0}\left(S, 1, \tilde{\mu}_{t-1}\right)\right]
$$

where $\tilde{\mu}_{t-1}$ corresponds to the Lagrange multiplier associated with equation (NKPC). However, the loss as well as inflation are close to their counterpart in the absence of cost push shock, thus:

$$
\left.\mathcal{L}_{t}=\frac{1}{1-\beta(1-\gamma)}\left[\pi^{2}+\beta \gamma L_{t_{l}}^{0}(S, 1)\right]\right]+o(\sigma)
$$

where $L_{t_{l}}^{0}(S, 1)$ is the loss of the conservative central banker in the absence of cost-push shock when mimicking the benevolent central banker's policy. Therefore, for $\sigma$ small enough, there exists an inflation level, $\tilde{\pi}$ (may be greater than the one in the absence of cost-push shocks) such that the incentive constraint of the conservative central banker is satisfied.

The benevolent central banker. As signaling may require a (infinitesimally) larger inflation level, as long as $\bar{q}$ is strictly larger than zero, it is always possible to find a smaller threshold such that the benevolent central banker incentive constraint is satisfied by continuity and because this constraint is more and more slack with $q$ (while $q$ does not modify the IC of the conservative type).

Finally, for small enough shocks and assuming that $\bar{q}>0$, we prove that the two incentive constraints are satisfied simultaneously. Our main result is hence robust to small cost push shocks. 
The following proposition formalizes these insights result as follows:

Proposition 10. If cost push shocks are small enough and if the threshold $\bar{q}$ is strictly positive in the absence of cost push shocks, then there exists a threshold, $\tilde{q}>0$, and an inflation level $\tilde{\pi}$ such that:

- If the beliefs are sufficiently biased toward the conservative central banker, $q<\tilde{q}$, then the equilibrium is separating and the benevolent central banker sets the positive inflation level before the liquidity trap $\tilde{\pi}$.

- Otherwise, $q>\tilde{q}$, the equilibrium is pooling and inflation prior to the liquidity trap is 0 .

Obviously, if shocks are sufficiently large, then the incentive to deviate for the conservative central banker will always dominate the gain from forward guidance and hence separating will emerge before the trap occurs.

\section{$7 \quad$ Further discussion}

Contracting with the central banker. The main problem associated with the implementation of the Ramsey policy is then the alignment of the central banker's preferences with welfare. And so, rather than relying on inefficient policies such as signaling through inflation, one can argue that private agents can also contract on the central banker's compensation to provide commitment ability. Such a performance contract has been shown to implement the second best policy in the absence of liquidity traps (see Walsh, 1995; Svensson, 1997).

In this paragraph, we mention two reasons that may prevent such contracting.

To start with, note that if the state-space of contracts is sufficiently rich and if agents can commit to contracts, then the Ramsey policy can be implemented whatever the preferences of agents. In particular, the society can write a contract with the central banker to reveal its type and that can also implement forward guidance once the trap is over. In particular, such contracts have to be history dependent to sustain forward guidance.

However, even when we restrict attention to a smaller set of contracts that are not history dependent and that are measurable only on current variables (e.g. current inflation and output gap), a transfer that would give an inflation bias to the conservative central banker would provide him with an ability to commit.

We argue that two caveats may prevent the implementation of such a contract. The first one is that the central banker's discount rate can be low. As a result, giving an inflation bias to him will not increase the ability to commit but just translates in higher inflation both 
before and after the trap. Under some conditions, this can be optimal (when the trap is sufficiently likely) but not always.

The second caveat is that the responsiveness of the central banker to transfers may be also private information. More precisely, his preferences can be of the form:

$$
\Gamma \pi_{t}^{2}
$$

where $\Gamma$ is private information (it does not appear in any of the policy implemented by the conservative central banker). As a result, when augmented with a transfer that creates an inflation bias, these preferences are:

$$
\Gamma \pi_{t}^{2}+\lambda\left(y-y^{*}\right)^{2}
$$

In the end, the precise weight on output $\lambda / \Gamma$ is private information.

\section{How to implement monetary policy taking into account the signaling issue? A} non-linear Taylor rule could implement a policy that signals the commitment type of monetary policy. The reaction to the natural interest rate should be more than one for one when the natural rate is low, and therefore the probability of the ZLB is high, so as to raise inflation in such a situation.

Probability of future ZLB. Our benchmark model rules out the possibility of future subsequent liquidity traps. Such an assumption makes tractable the signaling issue as central bankers are ultimately separated. Yet, traps can still occur with probability in the future and they also can vary in terms of intensity or length. In this case, our reasoning can be extended as follows: central bankers will only be naturally separated after any trap that requires stronger forward guidance than average. Otherwise, when the economy experiences short and mild traps, conservative central bankers can still be tempted to imitate the benevolent central bankers until a sufficiently long and strong trap occurs.

\section{Conclusion.}

This paper argues that raising the inflation is a way to solve an asymmetric information problem concerning the central banker's motive to stabilize inflation: a central banker that stabilizes inflation because of his ability to commit has an incentive to distinguish himself from a conservative central banker acting under discretion. Our main result is that signaling can be achieved solely by raising inflation. Quantitatively, we show that the benevolent central 
banker can optimally raise the inflation rate above standard inflation targets followed by central banks around the world. 


\section{References}

Andrade, P., G. Gaballo, E. Mengus, And B. Mojon (2015): "Forward Guidance and Heterogeneous Beliefs," Working Paper 573, Banque de France.

Aruoba, S. B. And F. Schorfheide (2013): "Macroeconomic dynamics near the ZLB: a tale of two equilibria," Working Papers 13-29, Federal Reserve Bank of Philadelphia.

Backus, D. AND J. Driffill (1985): "Inflation and Reputation," American Economic Review, 75, 530-38.

BALl, L. M. (2014): "The Case for a Long-Run Inflation Target of Four Percent," .

BARro, R. J. (1986): "Reputation in a model of monetary policy with incomplete information," Journal of Monetary Economics, 17, 3-20.

Barsky, R., A. Justiniano, And L. Melosi (2014): "The Natural Rate of Interest and Its Usefulness for Monetary Policy," American Economic Review, 104, 37-43.

Bassetto, M. (2015): "Forward Guidance: Communication, Commitment, or Both?" .

Blanchard, O. J., G. Dell'Ariccia, and P. Mauro (2010): "Rethinking Macroeconomic Policy," Journal of Money, Credit and Banking, 42, 199-215.

Bodenstein, M., J. Hebden, And N. Ricardo (2012): "Imperfect credibility and the zero lower bound," Journal of Monetary Economics, 59, 135-149.

Chari, V. V. And P. J. Kehoe (1990): "Sustainable Plans," Journal of Political Economy, $98,783-802$.

Coibion, O., Y. Gorodnichenko, and J. Wieland (2012): "The Optimal Inflation Rate in New Keynesian Models: Should Central Banks Raise Their Inflation Targets in Light of the Zero Lower Bound?" Review of Economic Studies, 79, 1371-1406.

Del Negro, M., M. Giannoni, and C. Patterson (2013): "The Forward Guidance Puzzle," Tech. rep., Federal Reserve Bank of New York, staff Report No. 574.

Eggertsson, G. B. And M. Woodford (2003): "The Zero Bound on Interest Rates and Optimal Monetary Policy," Brookings Papers on Economic Activity, 34, 139-235.

McKay, A., E. Nakamura, And J. Steinsson (2015): "The Power of Forward Guidance Revisited," NBER Working Papers 20882, National Bureau of Economic Research, Inc. 
Rogoff, K. (1985): "The Optimal Degree of Commitment to an Intermediate Monetary Target," The Quarterly Journal of Economics, 100, 1169-89.

Rosengren, E. S. (April 16,2015): "Changing Economic Relationships: Implications for Monetary Policy and Simple Monetary Policy Rules," Chatham House, speech.

Sleet, C. And S. Yeltekin (2007): "Recursive monetary policy games with incomplete information," Journal of Economic Dynamics and Control, 31, 1557-1583.

Svensson, L. E. O. (1997): "Optimal Inflation Targets, \&quot;Conservative\&quot; Central Banks, and Linear Inflation Contracts," American Economic Review, 87, 98-114.

Walsh, C. E. (1995): "Optimal Contracts for Central Bankers," American Economic Review, $85,150-67$.

Werning, I. (2012): "Managing Liquidity Trap: Monetary and Fiscal Policy," Tech. rep., MIT.

Woodford, M. (2003): Interest and Prices: Foundations of a Theory of Monetary Policy, Princeton University Press. 


\section{A The effect of belief on commitment.}

Figure 1 plots inflation, the output gap and the nominal interest rate when a 12-period liquidity trap hits the economy in period 1. Thin, thigh, dashed and thick with crosses denote the dynamics of the economy under the benevolent central banker if the prior beliefs on the commitment ability of the central banker, $q$, are respectively $1,0.5,0.05$ and 0 .
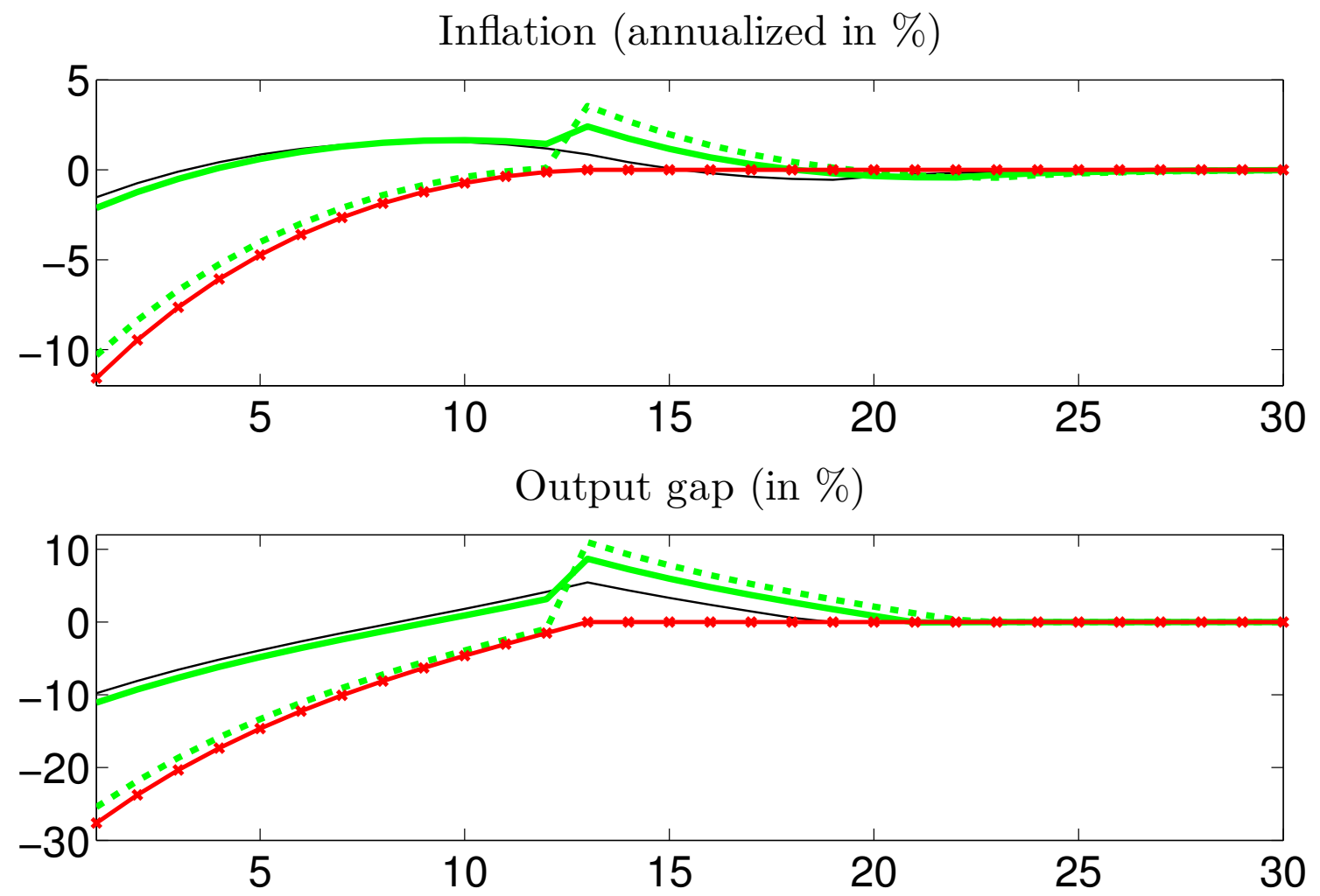

Nominal interest rate (annualized in \%)

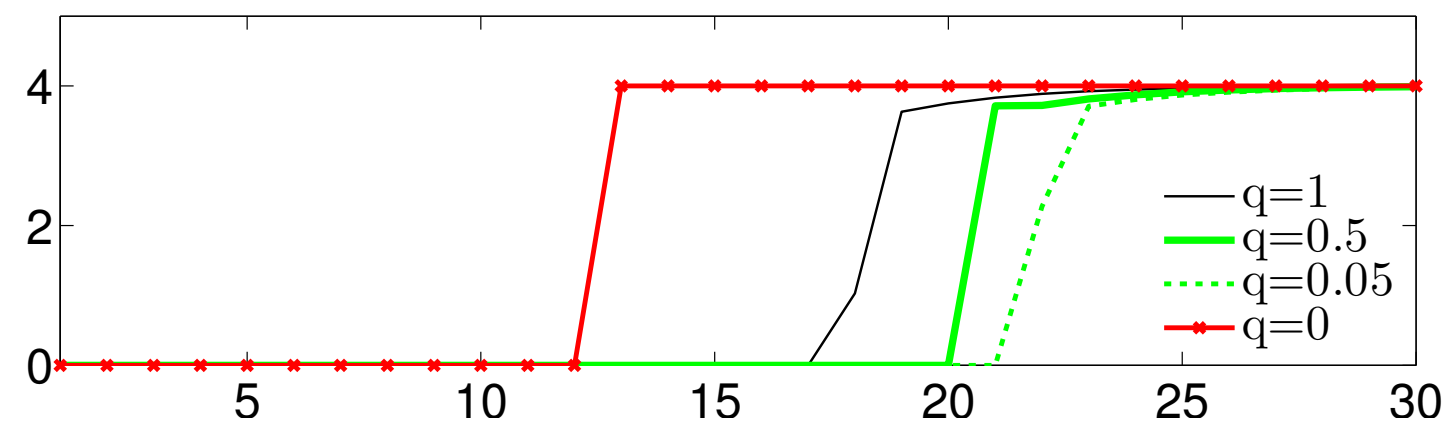

Figure 1 - Liquidity trap and prior beliefs

When $q=1$, the dynamics of the economy is not affected by imperfect information and the efficiency of the forward guidance is maximal. Inflation is close to 0 and the boom needed after the liquidity trap is relatively small. The forward guidance lasts 5 periods. In the contrary, when private agents believe that the central banker is the conservative one, the forward guidance is useless and the dynamics is the discretionary one. The recession is deep and long lasting. For intermediate cases, the forward guidance is longer than in the commitment case $(q=1)$ leading to a large boom after the trap ended.The efficiency of the forward guidance 
is quite effective except when beliefs are very biased toward the conservative central banker. At the limit, when $q$ is very close to 0 , the cost of the forward guidance after the trap dominates the gain during the trap and the duration of the forward guidance diminishes down to 0 .

\section{B Proofs}

\section{B.1 Proof of Proposition 1.}

Given the Ramsey allocation, we can observe that inequality (5) is neither satisfied for $\lambda_{D}=0$ nor for $\lambda_{D}=\infty$. In the former case positive inflation after the liquidity trap is impossible to sustain. In the latter case, asymptotic zero inflation and zero output gap after the liquidity trap leads to an infinite loss on the left-hand-side of inequality 5, while the best deviation leads to finite loss (right hand side).

As a result, the set of $\lambda_{D}$ for which inequality (5) holds is bounded. In addition, as inequality (5) is a weak inequality, this set is closed and so it is a compact, if non-empty, or it is the empty set. In the former case, there exists a lower bound of this set that we denote by $\underline{\lambda}_{D}$. Then, for any $\lambda_{D}<\underline{\lambda}_{D}$, inequality (5) is not satisfied. Symmetrically, we can define $\bar{\lambda}_{D}$ as the supremum of $\lambda_{D}$ values satisfying inequality (5).

\section{B.2 Proof of Proposition 2.}

Solution to problem 3 . The first order conditions until a trap occurs are:

$$
\begin{aligned}
& \pi_{t}+\mu_{t}-\mu_{t-1}-\pi_{t} \sum_{\tau=s}^{t} \beta^{t-\tau}(1-\gamma)^{t-\tau} \eta=0, \\
& \lambda\left(y_{t}-y^{*}\right)-\kappa \mu_{t}=0 .
\end{aligned}
$$

where $\eta$ is the Lagrange multiplier associated with the revelation constraint (REV). We crucially assume that the optimization takes place and constraint (REV) binds at $t=-\infty$. Therefore, the stationary solution must solve:

$$
\begin{aligned}
& \pi\left(1-\frac{1}{1-\beta(1-\gamma)} \eta\right)=0 \\
& \lambda\left(y-y^{*}\right)-\kappa \mu=0
\end{aligned}
$$

As a result, $(1-\beta(1-\gamma))=\eta$. This also implies that $\pi_{t}$ before the trap is constant and determined by (REV), that can be rewritten as follows:

$$
\pi^{2} \geq \beta \gamma\left[L_{t_{l}}^{0}(S, 0)-L_{t_{l}}^{0}(S, 1)\right]
$$

where the index $t_{l}$ means that the loss is computed during the trap.

During the trap the system of first order conditions is:

$$
\begin{aligned}
& \pi_{t}+\mu_{t}-\mu_{t-1}-\frac{\nu_{t-1}}{\beta \sigma}-\pi_{t} \sum_{\tau=-\infty}^{t_{l}} \beta^{t-\tau}(1-\gamma)^{t_{l}-\tau} \gamma \eta=0, \\
& \lambda\left(y_{t}-y^{*}\right)-\kappa \mu_{t}+\nu_{t}-\frac{1}{\beta} \nu_{t-1}=0 \\
& \frac{1}{\sigma} \nu_{t}+\zeta_{t}=0 .
\end{aligned}
$$


Substituting $\eta$ by its value yields:

$$
\begin{aligned}
& \pi_{t}\left(1-\beta^{t-t_{l}-1} \gamma\right)+\mu_{t}-\mu_{t-1}-\frac{\nu_{t-1}}{\beta \sigma}=0, \\
& \lambda\left(y_{t}-y^{*}\right)-\kappa \mu_{t}+\nu_{t}-\frac{1}{\beta} \nu_{t-1}=0, \\
& \frac{1}{\sigma} \nu_{t}+\zeta_{t}=0 .
\end{aligned}
$$

After the trap, the system of first order conditions is as under perfect information:

$$
\begin{aligned}
& \pi_{t}+\mu_{t}-\mu_{t-1}-\frac{\nu_{t-1}}{\beta \sigma}=0, \\
& \lambda\left(y_{t}-y^{*}\right)-\kappa \mu_{t}+\nu_{t}-\frac{1}{\beta} \nu_{t-1}=0, \\
& \frac{1}{\sigma} \nu_{t}+\zeta_{t}=0
\end{aligned}
$$

This proves that inflation is more negative during the trap and the forward guidance is weaker compared to the perfect information case (TBC).

Sustainability. After the liquidity trap, the central banker type is unrevealed. Therefore, Proposition 1 applies and according to assumption 3, the benevolent central banker will not choose to deviate. In numerical applications, we check that this assumption is well satisfied.

If the inflation level set by the benevolent central banker is below the steady state level of inflation under discretion, then the benevolent central banker would like to raise the inflation rate further. Compared to the sustainability constraint for the benevolent central banker in full information, the sustainability constraint, equation (4) is slacker. The deviation is more costly as inflation expectations are higher due to the positive inflation level before the trap and the loss under the Perfect Bayesian equilibrium is lower has a higher inflation level leads to lower loss. This would lead to an increase in the deviation loss.

If the signaling inflation level is above the steady state level of inflation under discretion (for instance, $y^{*}$ is close to 0 ), the benevolent central banker would like to lower the level of inflation but it is impossible as at the equilibrium, constraint (REV) is binding and hence a lower inflation would lead to pooling.

\section{B.3 Proof of Proposition 3}

Dynamics. For any period before the trap, the ZLB has not hit, and so, $\zeta_{t}=\nu_{t}=0$. The set of equations is then:

$$
\pi_{t}+\frac{\lambda}{\kappa}\left(y_{t}-y_{t-1}\right)=0
$$

associated with (NKPC) and (EE). In a timeless perspective, this implies that $\pi_{t}=0$ and $y_{t}=-\frac{\kappa}{\beta \gamma} \pi_{l}$. The resulting value for $\mu_{t}$ is $\mu_{t}=-\frac{\lambda}{\kappa}\left(\frac{\kappa}{\beta \gamma} \pi_{l}+y^{*}\right)$. We denote by $\tilde{\mu}_{t}$ the modified Lagrange multiplier $\tilde{\mu}_{t}=\tilde{m} u_{t}+\frac{\lambda}{\kappa} y^{*}$.

Lemma 11. If $\sigma \kappa / \lambda<1$, for all $t>l, \pi_{t}$ and $y_{t}$ are strictly positive and $\pi_{l}$ and $y_{l}$ are strictly negative, for any $q$. 
Proof. We first show that after the trap and after leaving the ZLB, $\pi_{t}$ and $y_{t}$ have the same sign and converge to 0 . Indeed, they solve, jointly with $\tilde{\mu}_{t}$ the following problem:

$$
\begin{array}{r}
\pi_{t}=\beta \pi_{t+1}+\kappa y_{t} \\
\lambda y_{t}-\kappa \tilde{\mu}_{t}=0 \\
\pi_{t}+\tilde{\mu}_{t}-\tilde{\mu}_{t-1}=0
\end{array}
$$

This allows to solve for $\pi_{t}$ and $y_{t}$ given an initial value for $\tilde{\mu}_{t-1} \cdot \tilde{\mu}_{t}$ follows a second order linear difference equation:

$$
\beta \tilde{\mu}_{t+1}-\left(\beta+\frac{\kappa^{2}}{\lambda}+1\right) \tilde{\mu}_{t}+\tilde{\mu}_{t-1}=0
$$

whose (bounded) solution is:

$$
\tilde{\mu}_{t}=\Gamma_{3} \tilde{\mu}_{t-1}
$$

where $\Gamma_{3}=\left[\frac{1}{2}+\frac{\kappa^{2}}{2 \beta \lambda}+\frac{1}{2 \beta}\right]-\sqrt{\left[\frac{1}{2}+\frac{\kappa^{2}}{2 \beta \lambda}+\frac{1}{2 \beta}\right]^{2}-1} \in(0,1)$. As a consequence, $\tilde{\mu}_{t}$ monotonically converges to 0 , which implies that $\pi_{t}$ and $y_{t}$ converge to 0 as well. In addition, either $\tilde{\mu}_{t}$ is decreasing and positive, and so are $\pi_{t}$ and $y_{t}$, or $\tilde{\mu}_{t}$ is increasing and negative, and so are $\pi_{t}$ and $y_{t}$.

After the trap not at the ZLB, first period:

$$
\begin{array}{r}
\pi_{t}=\beta \pi_{t+1}+\kappa y_{t} \\
\lambda y_{t}-\kappa \tilde{\mu}_{t}-\frac{\sigma}{\beta} q \zeta_{t-1}=0 \\
\pi_{t}+\tilde{\mu}_{t}-q \tilde{\mu}_{t-1}-q \frac{\zeta_{t-1}}{\beta}=0
\end{array}
$$

We use the solution of the first equations to substitute $\pi_{t+1}$ by something proportional to $\tilde{\mu}_{t}$, and we find $\pi_{t}$, $y_{t}$ and $\tilde{\mu}_{t}$ as functions of $\zeta_{t-1}$ and $\tilde{\mu}_{t-1}$.

This is the same equations than before except that there is now a constant due to the past Lagrange multiplier associated with past ZLB. $\tilde{\mu}_{t}$ follows a second order linear difference equation:

$$
\beta \tilde{\mu}_{t+1}-\left(\beta+\frac{\kappa^{2}}{\lambda}+1\right) \tilde{\mu}_{t}+q \tilde{\mu}_{t-1}=-\frac{q}{\beta}\left(\frac{\sigma \kappa}{\lambda}-1\right) \zeta_{t-1}
$$

Thus, the solution should be as follows:

$$
\tilde{\mu}_{t}=q\left[\Gamma_{2} \zeta_{t-1}+\Gamma_{3} \tilde{\mu}_{t-1}\right]
$$

where $\Gamma_{2}=\frac{\beta+\frac{\kappa^{2}}{\lambda}+1}{\beta}\left(\frac{\sigma \kappa}{\lambda}-1\right)$. When, $\sigma \kappa / \lambda<1$ (see Werning (2012)), $\Gamma_{2}<1$.

In period $l$, the set of equations is:

$$
\begin{array}{r}
\pi_{l}=\beta q \pi_{l+1}+\kappa y_{l}, \\
\lambda y_{l}-\kappa \tilde{\mu}_{l}-\sigma \zeta_{l}=0, \\
\pi_{l}+\tilde{\mu}_{l}=0 .
\end{array}
$$

Equations (29) and (31) prove that inflation is always positive after the trap (for $t>l+2$ ) if only if $\mu_{l+1}$ is positive. Then, equation (36) shows that it depends on the value of $\tilde{\mu}_{l}$. We now prove that $\tilde{\mu}_{l}$ is positive, i.e. $\pi_{l}$ is negative. 
We combine equation (NKPC) at the ZLB period and equations giving $\pi_{l+1}$ and $y_{l}$ with respect to $\pi_{l}$ and $\zeta_{l}$ and we obtain:

$$
\left[1+\frac{\kappa^{2}}{\lambda}+\beta q^{2}\left(1-\Gamma_{3}\right)\right] \pi_{l}=\left[\frac{\sigma \kappa}{\lambda}+\frac{\beta q^{2}}{\beta}-q \Gamma_{2}\right] \zeta_{l}
$$

Assuming that $\frac{\sigma \kappa}{\lambda}<1$, and remarking that $\zeta_{l}$ is the Lagrange multiplier associated with (ZLB) and hence is negative, we thus obtain that $\pi_{l}<0$. Equation (38) shows that $y_{l}$ is also negative in such a case.

Losses. The envelope condition of the two loss functions with respect to q leads to:

$$
\frac{\partial L_{t_{l}}(P, q)}{\partial q}=-\beta \mu_{l} \pi_{l+1}-\nu_{l}\left(y_{l+1}+\frac{\pi_{l+1}}{\sigma}\right)
$$

where $l=t_{l}$ is the period at which the economy is facing the shock. $\mu_{l}=-\pi_{l}>0, \nu_{l}=-\sigma \zeta_{l}>0$. Variables taken at time $l+1$ correspond to the situation if the central banker is benevolent. Thus, $\pi_{l+1}>0$ as well as $y_{l+1}>0$. Finally, these derivatives are negative, which yields our result. We can observe that the loss of the conservative central banker is proportional to the square of $\pi_{l}$, thus, inflation is increasing in $q$ during the liquidity trap. The same computation proves that $L_{t_{l}}^{0}(P, q)$ is decreasing in $q$.

\section{B.4 Proof of Theorem 4 .}

The benevolent central banker prefers the separating equilibrium over the pooling equilibrium if:

$$
\frac{\pi^{2}+\lambda\left(y-y^{*}\right)^{2}+\beta \gamma L_{t_{l}}(S, 1)}{1-\beta(1-\gamma)} \leq \frac{\pi_{p}^{2}+\lambda\left(y_{p}-y^{*}\right)^{2}+\beta \gamma L_{t_{l}}(P, q)}{1-\beta(1-\gamma)}
$$

where $\pi$ and $y$ (resp. $\pi_{p}$ and $y_{p}$ ) are the output-gap and inflation in the separating (pooling resp.) equilibrium before the liquidity trap. One can observe that $\pi_{p}=0$ because it corresponds to the best policy of the conservative central banker. The (NKPC) leads to $y=\frac{1-\beta(1-\gamma)}{\kappa} \pi+y_{p}(q)$ and $y_{p}=-\frac{\beta \gamma}{\kappa} \pi_{l}$. This inequality can be rewritten as:

$$
\beta \gamma\left(L_{t_{l}}(P, q)-L_{t_{l}}(S, 1)\right) \geq \pi^{2}+\lambda\left[\left(y-y^{*}\right)^{2}-\left(y_{p}-y^{*}\right)^{2}\right]
$$

The conservative central banker prefers imitating the benevolent central banker if equation (REV) is violated thus the two relevant incentive constraints are:

$$
\begin{aligned}
\beta \gamma\left(L_{t_{l}}(P, q)-L_{t_{l}}(S, 1)\right) \geq & \pi^{2}+\lambda\left[\left(y-y^{*}\right)^{2}-\left(y_{p}-y^{*}\right)^{2}\right] \\
& \pi^{2} \geq \beta \gamma\left[L_{t_{l}}^{0}(S, 0)-L_{t_{l}}^{0}(S, 1)\right]
\end{aligned}
$$

No liquidity trap. When $\gamma=0$, the two conditions (45) and (44) are equalities when both types follow their optimal policies, thus, they are indifferent between pooling and separating equilibria. Thus, the equilibrium is independent of the prior, $q$. 
Liquidity trap. Ex ante output gains due to the anticipation of ZLB dominate the steady state output inefficiency:

$$
\begin{array}{r}
\gamma\left(L_{t_{l}}(P, q)-L_{t_{l}}(S, 1)\right) \geq(1-\gamma)\left[\left(1+\frac{\lambda}{\kappa}(1-\beta(1-\gamma)) \pi^{2}+2 \frac{\lambda}{\kappa}(1-\beta(1-\gamma)) \pi\left(y_{p}-y^{*}\right)\right]\right. \\
\pi^{2} \geq \beta \gamma\left[L_{t_{l}}^{0}(S, 0)-L_{t_{l}}^{0}(S, 1)\right]
\end{array}
$$

Note that, if $y^{*}$ sufficiently low, as $y_{p}$ decreases with $\mathrm{q}, \pi^{2}+\lambda\left[\left(y-y^{*}\right)^{2}-\left(y_{p}-y^{*}\right)^{2}\right]$ increases with $q$. This allows to conclude as follows. Either $L_{t_{l}}(0)$ is such that (46) holds and, then, there exists $\tilde{q}$ such that, for $q \leq \bar{q}$, (46) holds or $\bar{q}=0$. Finally, note that $\bar{q}$ is strictly below 1 as $L_{t_{l}}(S, 1)>L_{t_{l}}(P, 1)$.

\section{B.5 Proof of Proposition 5.}

Suppose that $\lambda=0$. Combining (7) and (8) yields

$$
\left(L_{t_{l}}(P, q)-L_{t_{l}}(S, 1)\right) \geq\left[L_{t_{l}}^{0}(S, 0)-L_{t_{l}}^{0}(S, 1)\right],
$$

which implies that $\bar{q}=0$ and there exists no separating equilibrium.

Conversely, suppose that $\lambda>0$. Combining (7) and (8) yields:

$$
\beta \gamma\left(L_{t_{l}}^{0}(S, 1)-L_{t_{l}}^{0}(S, 0)+L_{t_{l}}(P, 0)-L_{t_{l}}(S, 1)\right) \geq \lambda\left[\left(y-y^{*}\right)^{2}-\left(y_{p}-y^{*}\right)^{2}\right]
$$

When $y^{*}$ goes to infinity, this inequality becomes: $\beta \gamma\left(L_{t_{l}}(P, 0)-L_{t_{l}}(S, 1)\right) \geq 0$, which is always strictly satisfied. Finally, note that we also have $\beta \gamma\left(L_{t_{l}}(P, 0)-L_{t_{l}}(P, 1)\right) \geq 0$ and, as $L_{t_{l}}(P, q)$ is decreasing in $q$, this implies that $\bar{q}$ is strictly positive, when $y^{*}$ is sufficiently large.

The second part of the proposition results from the fact that the derivative of the right hand term of (7) with respect to $y^{*}$ is negative.

\section{B.6 Proof of Corollary 6.}

When $q \leq \bar{q}$, the equilibrium is separating, inflation under the benevolent central banker is strictly positive (except if $q=0$ ) and given by the IC of the conservative central banker (8). Hence, inflation is increasing with $q$ and reaches its maximum for $q=\bar{q}$.

$\pi$ is determined as follows:

$$
\pi^{2} \geq \beta \gamma\left[L_{t_{l}}^{0}(S, 0)-L_{t_{l}}^{0}(S, 1)\right] .
$$

Thus, $\pi$ is increasing in $\gamma$. As $L_{t_{l}}^{0}(S, 0)-L_{t_{l}}^{0}(S, 1)$ increases with $r, \pi$ increases with $r$ as well.

\section{B.7 Proof of Proposition 7.}

We want to determine the derivative of

$$
\left[\left(y-y^{*}\right)^{2}-\left(y_{p}-y^{*}\right)^{2}\right]=\left(y-y_{p}\right)\left(y+y_{p}-2 y^{*}\right) .
$$

Using (NKPC), we obtain:

$$
\begin{aligned}
& y-y_{p}(q)=\frac{1-\beta(1-\gamma)}{\kappa} \pi-\frac{\beta \gamma}{\kappa}\left(\pi_{l}(1)-\pi_{l}(q)\right), \\
& y+y_{p}(q)-2 y^{*}=\frac{1-\beta(1-\gamma)}{\kappa} \pi-\frac{\beta \gamma}{\kappa}\left(\pi_{l}(1)+\pi_{l}(q)\right)-2 y^{*} .
\end{aligned}
$$


When $\gamma$ is close to 0 , the two expressions lead to:

$$
\begin{aligned}
& y-y_{p}(q)=\frac{1-\beta}{\kappa} A \gamma^{1 / 2}+o\left(\gamma^{1 / 2}\right), \\
& y+y_{p}(q)-2 y^{*}=-2 y^{*}+A^{2} \gamma+o(\gamma),
\end{aligned}
$$

with $A=\left(\beta\left[L_{t_{l}}^{0}(S, 0)-L_{t_{l}}^{0}(S, 1)\right]\right)^{1 / 2}$. Then:

$$
\left[\left(y-y^{*}\right)^{2}-\left(y_{p}-y^{*}\right)^{2}\right]=-2 A y^{*} \gamma^{1 / 2}+A^{2} \gamma+o(\gamma) .
$$

\section{B.8 Proof of Proposition 8.}

The incentive constraint for the benevolent central banker writes:

$$
\begin{aligned}
& \frac{\pi_{1}^{2}+\lambda\left(y_{1}-y^{*}\right)^{2}+\beta \gamma_{1} L_{t_{l}}(S, 1)}{1-\beta \Pi_{1}}+\beta\left(1-\gamma_{1}-\Pi_{1}\right) \frac{\pi_{2}^{2}+\lambda\left(y_{2}-y^{*}\right)^{2}+\beta \gamma_{2} L_{t_{l}}(S, 1)}{1-\beta \Pi_{2}} \geq \cdots \\
& \ldots \frac{\lambda\left(y_{p}-y^{*}\right)^{2}+\beta \gamma_{1} L_{t_{l}}(P, q)}{1-\beta \Pi_{1}}+\beta\left(1-\gamma_{1}-\Pi_{1}\right) \frac{\lambda\left(y_{p}-y^{*}\right)^{2}+\beta \gamma_{2} L_{t_{l}}(P, q)}{1-\beta \Pi_{2}}
\end{aligned}
$$

The incentive constraint for the conservative central banker writes:

$$
\frac{\pi_{1}^{2}+\gamma_{1} L_{t_{l}}^{0}(S, 1)}{1-\beta \Pi_{1}}+\beta\left(1-\gamma_{1}-\Pi_{1}\right) \frac{\pi_{2}^{2}+\gamma_{2} L_{t_{l}}^{0}(S, 1)}{1-\beta \Pi_{2}} \geq \frac{\beta \gamma_{1}}{1-\beta \Pi_{1}} L_{t_{l}}^{0}(S, 0)+\beta\left(1-\gamma_{1}-\Pi_{1}\right) \frac{\beta \gamma_{2}}{1-\beta \Pi_{2}} L_{t_{l}}^{0}(S, 0)
$$

Similarly:

$$
\frac{\pi_{2}^{2}+\gamma_{2} L_{t_{l}}^{0}(S, 1)}{1-\beta \Pi_{2}}+\beta\left(1-\gamma_{2}-\Pi_{2}\right) \frac{\pi_{1}^{2}+\gamma_{1} L_{t_{l}}^{0}(S, 1)}{1-\beta \Pi_{1}} \geq \frac{\beta \gamma_{2}}{1-\beta \Pi_{2}} L_{t_{l}}^{0}(S, 0)+\beta\left(1-\gamma_{2}-\Pi_{2}\right) \frac{\beta \gamma_{1}}{1-\beta \Pi_{1}} L_{t_{l}}^{0}(S, 0)
$$

These two inequalities hold with equality at the optimum. Multiplying the second one by $\beta\left(1-\gamma_{1}-\Pi_{1}\right)$ and subtracting it from the first one, we can then simplify the expression that we find, and divide it by $\left(1-\beta^{2}\left(1-\gamma_{2}-\Pi_{2}\right)\left(1-\gamma_{1}-\Pi_{1}\right)\right)$. We then obtain:

$$
\pi_{1}^{2}=\beta \gamma_{1}\left(L_{t_{l}}^{0}(S, 0)-L_{t_{l}}^{0}(S, 1)\right) \text { and } \pi_{2}^{2}=\beta \gamma_{2}\left(L_{t_{l}}^{0}(S, 0)-L_{t_{l}}^{0}(S, 1)\right)
$$

which yields the result. 